\title{
Measurements of top quark properties with the CMS experiment
}

\section{Joscha Knolle* on behalf of the CMS Collaboration}

DESY, Hamburg, Germany

E-mail: joscha.knolle@cern.ch

Measurements of properties of the top quark with the CMS experiment at the CERN LHC are presented. Top quark pair production events at $\sqrt{s}=13 \mathrm{TeV}$ are used to measure the top quark Yukawa coupling, to study the spin correlation between the top quarks, and to extract the forwardbackward asymmetry in quark-antiquark initiated production. A combination of the measurements of $\mathrm{W}$ boson polarization in top quark pair and single top quark production events at $\sqrt{s}=8 \mathrm{TeV}$ performed by the ATLAS and CMS experiments is shown. A first direct measurement of the elements of the CKM matrix involving the top quark is performed in single top quark production events at $\sqrt{s}=13 \mathrm{TeV}$.

The Eighth Annual Conference on Large Hadron Collider Physics-LHCP2020

25-30 May, 2020

online

${ }^{*}$ Speaker 


\section{Introduction}

The CMS experiment [1] exploits the vast number of top quark pair (t $\mathrm{t})$ and single top quark production events in proton-proton collisions at the CERN LHC to perform measurements of various properties of the top quark and its decay products. In this contribution, the latest results are presented and compared to standard model (SM) predictions.

\section{Results}

Top quark Yukawa coupling The Yukawa coupling of the top quark, $g_{t}$, contributes to tt production via virtual Higgs boson diagrams. Its effect can be probed in distributions of the invariant mass of the tit system, $M(\mathrm{t} \overline{\mathrm{t}})$, and the rapidity difference between top quark and antiquark, $\Delta y(\mathrm{t} \overline{\mathrm{t}})$.

The first measurement of $g_{\mathrm{t}}$ in $\mathrm{t} \overline{\mathrm{t}}$ events was performed in the lepton+jets final state using $35.8 \mathrm{fb}^{-1}$ of data collected at $\sqrt{s}=13 \mathrm{TeV}$ in 2016 [2]. A template fit to the reconstructed $M(\mathrm{t} \overline{\mathrm{t}})$ and $\Delta y(\mathrm{t} \mathrm{t})$ distributions in categories of jet multiplicity is used to extract $g_{\mathrm{t}}$.

In a recent measurement, $g_{\mathrm{t}}$ was measured in the dilepton final state using $137 \mathrm{fb}^{-1}$ of data collected at $\sqrt{s}=13 \mathrm{TeV}$ between 2016 and 2018 [3]. A partial reconstruction of the $\mathrm{t}^{-1}$ system is performed to resolve the assignment of $\mathrm{b}$ jets to top quark and antiquark. The Yukawa coupling is then extracted from a template fit to the reconstructed $M(\mathrm{~b} \ell, \mathrm{b} \ell)$ and $\Delta y(\mathrm{~b} \ell, \mathrm{b} \ell)$ distributions.

The results of the two measurements are listed in Table 1 . They agree with each other as well as with extractions from Higgs boson measurements [4] and from a tttt̄ cross section measurement [5].

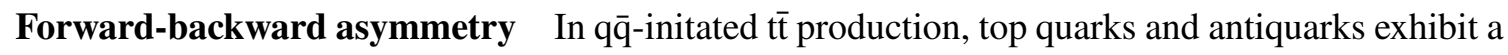
forward-backward asymmetry not present in gg- or gq-initated production, defined as:

$$
A_{\mathrm{FB}}=\frac{\sigma\left(\cos \theta^{*}>0\right)-\sigma\left(\cos \theta^{*}<0\right)}{\sigma\left(\cos \theta^{*}>0\right)+\sigma\left(\cos \theta^{*}<0\right)},
$$

with the angle $\theta^{*}$ between top quark and initial-state quark in the tit rest frame. The distributions of $\cos \theta^{*}, M(\mathrm{t} \overline{\mathrm{t}})$, and the normalized longitudinal momentum of the $\mathrm{t} \overline{\mathrm{t}}$ system, $x_{F}$, allow for a discrimination between $\mathrm{q} \overline{\mathrm{q}}$-initiated and other $\mathrm{t} \overline{\mathrm{t}}$ events. $A_{\mathrm{FB}}$ can then be extracted from the reconstructed $\mathrm{q} \overline{\mathrm{q}} \rightarrow \mathrm{t} \overline{\mathrm{t}}$ differential cross section as a function of $\cos \theta^{*}$.

A measurement of $A_{\mathrm{FB}}$ was performed in the lepton+jets final state using $35.9 \mathrm{fb}^{-1}$ of data collected at $\sqrt{s}=13 \mathrm{TeV}$ in 2016 [6]. The $\mathrm{t} \overline{\mathrm{t}}$ system is reconstructed in events with resolved and boosted topologies of the hadronic decay. From a template fit to the reconstructed $\cos \theta^{*}, M(\mathrm{t} \overline{\mathrm{t}})$, and $x_{F}$ distributions in categories of lepton flavour and charge as well as jet topology, $A_{\mathrm{FB}}$ is extracted. The result, listed in Table 2, is in agreement with the SM prediction [7] and the Tevatron result [8].

Table 1: Top quark Yukawa coupling results.

Table 2: Forward-backward asymmetry results.

\begin{tabular}{lll} 
measurement & $\boldsymbol{g}_{\mathbf{t}} / \boldsymbol{g}_{\mathbf{t}}^{\text {SM }}$ & \\
\hline $\mathrm{t} \bar{l}$ lepton+jets & $1.07_{-0.43}^{+0.34}$ & {$[2]$} \\
$\mathrm{t} \bar{t}$ dilepton & $1.16_{-0.35}^{+0.24}$ & {$[3]$} \\
\hline Higgs boson global fit & $0.98 \pm 0.14$ & {$[4]$} \\
$\mathrm{t} \bar{t} \bar{t}(95 \%$ CL) & $<1.7$ & {$[5]$}
\end{tabular}

\begin{tabular}{lll} 
measurement & $\boldsymbol{A}_{\mathbf{F B}}$ \\
\hline $\mathrm{t} \mathfrak{\mathrm { t }}$ lepton+jets & $0.048_{-0.092}^{+0.097}$ & {$[6]$} \\
\hline NNLO QCD prediction & $0.095 \pm 0.007$ & {$[7]$} \\
Tevatron combination & $0.128 \pm 0.025$ & {$[8]$}
\end{tabular}




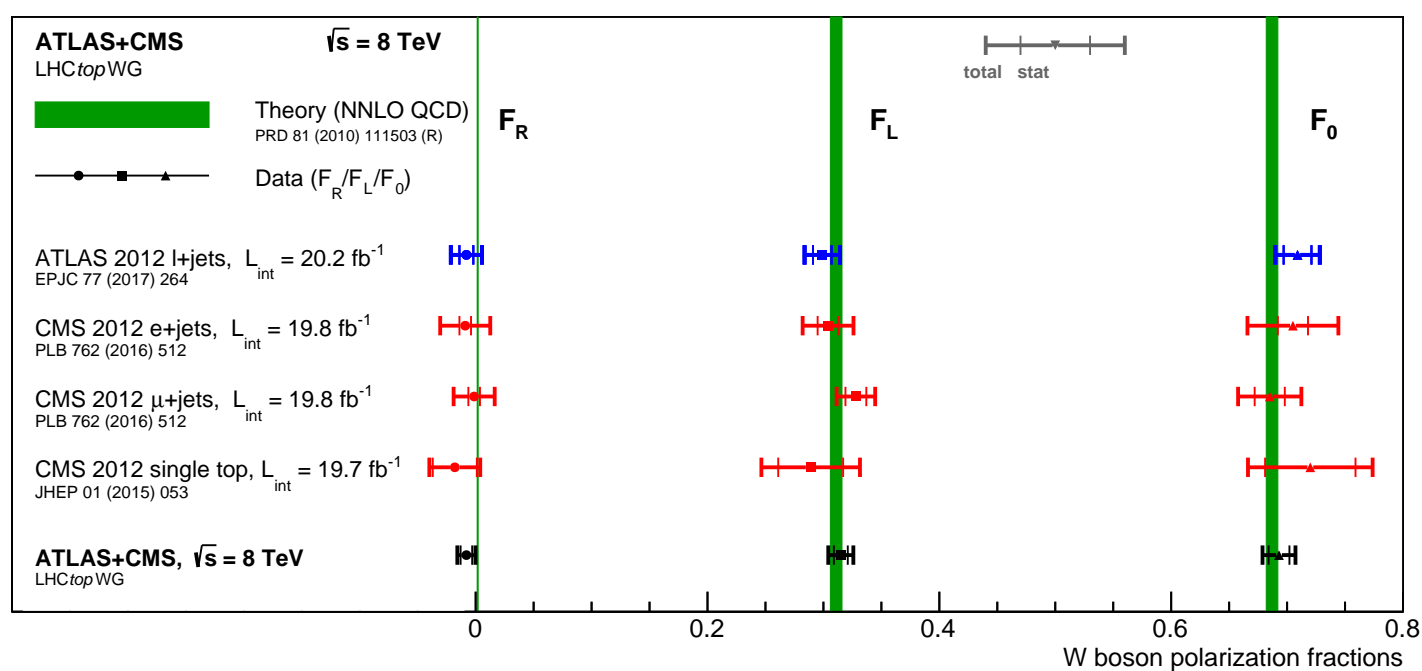

Figure 1: Summary of the measurements of $\mathrm{W}$ boson polarization fractions at the LHC [12].

W boson polarization in top quark decays The polarization of the $\mathrm{W}$ boson in top quark decays is governed by the $V-A$ structure of the weak interaction. The polarization fractions for longitudinal, left- and right-handed polarization, which satisfy $F_{0}+F_{L}+F_{R}=1$, are measured in tit or single top quark events from reconstructed $\cos \theta^{*}$ distributions where $\theta^{*}$ is the polarization angle between the direction of the lepton and the reversed direction of the $\mathrm{b}$ quark in the $\mathrm{W}$ boson rest frame.

The polarization fractions were measured using data collected at $\sqrt{s}=8 \mathrm{TeV}$ in 2012 by the ATLAS experiment in $t \bar{t}$ events [9], and by the CMS experiment both in $t \bar{t}$ and single top quark events [10,11]. Recently, a combination of these measurements was performed [12]. The results, summarized in Figure 1, are the most precise measurements of the W boson polarization fractions, and show good agreement with the SM predictions [13].

Elements of CKM matrix involving top quark In electroweak $t$-channel single top quark events, both production cross section and decay branching fraction of the top quark involve a CKM matrix element. The dominant contribution with $\mathrm{a} b$ quark from gluon splitting at the production vertex and a $\mathrm{b}$ quark in the decay has $\sigma \times \mathcal{B} \approx 220 \mathrm{pb}$. Contributions with a light quark in the production (decay) vertex are much rarer with $\sigma \times \mathcal{B} \approx 1 \mathrm{pb}(0.4 \mathrm{pb})$. For a direct measurement of the CKM matrix elements involving the top quark, the $b$ jet multiplicity as well as kinematic properties of jets identified as originating from the gluon splitting or the top quark decay are exploited.

The first direct extraction of the top quark CKM matrix elements was performed using $35.9 \mathrm{fb}^{-1}$ of data collected at $\sqrt{s}=13 \mathrm{TeV}$ in 2016 [14]. Multivariate discriminants are trained to separate single top quark events with $\mathrm{b}$ quarks both in production and decay from background events as well as from single top quark events with a light quark in either production or decay. A template fit is performed to the BDT output distributions in different jet multiplicity categories. Without assumptions on the number of quarks or the top quark decay channels, the CKM matrix elements are measured to be

$$
\left|V_{\mathrm{tb}}\right|=0.988 \pm 0.024 \quad \text { and } \quad\left|V_{\mathrm{td}}\right|+\left|V_{\mathrm{ts}}\right|=0.06 \pm 0.06 \text {. }
$$

The top quark decay width is found to be in good agreement with the SM expectation. 

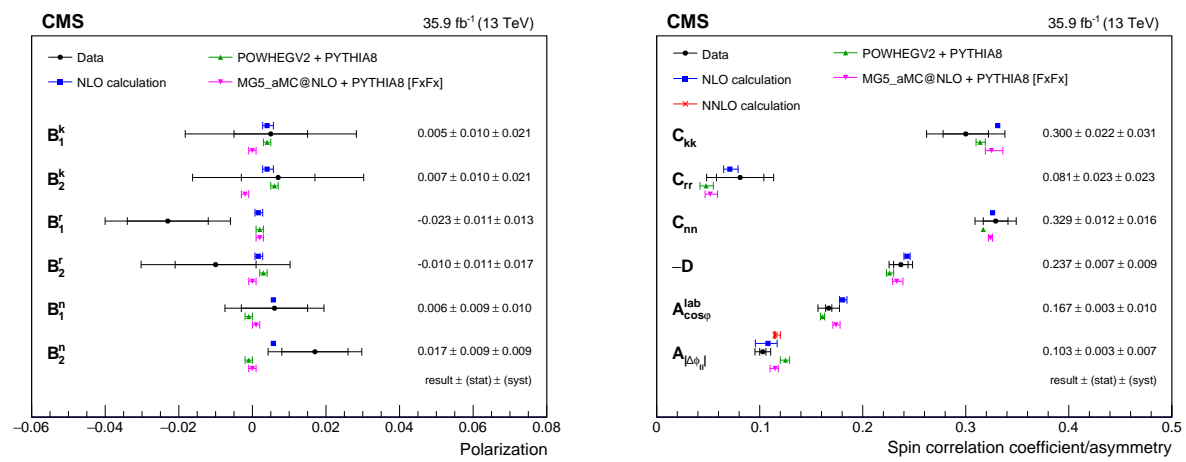

Figure 2: Results of the measurements of polarization and spin correlation coefficients [15].

Top quark pair spin correlations Due to its large decay width, the top quark spin information is preserved in angular distributions of its decay products. Such distributions can thus be used to measure top quark polarization coefficients as well as spin correlation coefficients of the $t \bar{t}$ system.

The CMS experiment performed a measurement of $t \bar{t}$ spin correlations in the dilepton final state, using $35.9 \mathrm{fb}^{-1}$ of data collected in 2016 [15]. The angular distributions of the leptons are unfolded to parton-level, and the polarization and spin correlation coefficients are extracted from the differential cross sections. A selection of the results is shown in Figure 2.

\section{Conclusions}

Several recent measurements of top quark properties have been presented. Profiting from the large number of $\bar{t}$ and single top quark production events at the LHC, the CMS experiment has achieved a high precision in its results. All results are in agreement with the SM predictions, and can be used to evaluate constraints on new physics models.

\section{References}

[1] CMS Collaboration, JINST 3 (2008) S08004.

[2] CMS Collaboration, Phys. Rev. D 100 (2019) 072007 [1907. 01590].

[3] CMS Collaboration, CMS-PAS-TOP-19-008, CERN 2020 [CDS : 2718943], superseded by: CMS Collaboration, 2009.07123 (submitted to Phys. Rev. D).

[4] CMS Collaboration. Eur. Phys. J. C 79 (2019) 421 [1809 . 10733].

[5] CMS Collaboration. Eur. Phys. J. C 80 (2020) 75 [1908. 06463].

[6] CMS Collaboration, JHEP 06 (2020) 146 [1912 . 09540].

[7] M. Czakon, P. Fiedler, \& A. Mitov, Phys. Rev. Lett. 115 (2015) 052001 [1411 . 3007].

[8] CDF \& DØ Collaborations, Phys. Rev. Lett. 120 (2018) 042001 [1709. 04894].

[9] ATLAS Collaboration, Eur. Phys. J. C 77 (2017) 264 [Erratum: Ibid. 79 (2019) 19] [1612 . 02577].

[10] CMS Collaboration, Phys. Lett. B 762 (2016) 512 [1605 . 09047].

[11] CMS Collaboration. JHEP 01 (2015) 053 [1410.1154].

[12] ATLAS \& CMS Collaborations, JHEP 08 (2020) 051 [2005. 03799].

[13] A. Czarnecki, J. Körner, \& J. Piclum, Phys. Rev. D 81 (2010) 111503 [1005 . 2625].

[14] CMS Collaboration, Phys. Lett. B 808 (2020) 135609 [2004.12181].

[15] CMS Collaboration, Phys. Rev. D 100 (2019) 072002 [1907.03729]. 